Pacific Journal of Mathematic 


\title{
TWO UNIFORM BOUNDEDNESS THEOREMS
}

\author{
JAMES D. STEIN JR.
}

A geodesically convex space is a metric space in which each two points can be connected by a unique segment (a path of minimal length). An affine transformation between two geodesically convex spaces is a map which takes segments into segments. It is shown that, if the domain is complete, a pointwise-bounded family of continuous affine transformations is uniformly bounded. Under a mild additional hypothesis, the following stronger theorem holds: if

$$
\mathscr{F}=\left\{T_{o} \mid A \in A\right\}
$$

is a pointwise-bounded family of affine transformatons and $T_{a}$ is continuous on a closed geodesically convex $S_{\alpha}$ with

$$
\bigcap_{A \in \alpha} S_{\alpha} \neq \varnothing
$$

then $\exists \alpha_{1}, \cdots, \alpha_{n}$ such that $\mathscr{T}$ is uniformly bounded on

$$
\bigcap_{k=1}^{n} S_{\alpha_{k}}
$$

Let $(X, d),\left(Y, d^{\prime}\right)$ be metric spaces, and $\mathscr{F}=\left\{T_{\alpha} \mid \alpha \in A\right\}$ a collection of maps from $X$ to $Y$. We say $\mathscr{F}$ is pointwise-bounded if, for fixed $x, y \in X, \sup \left\{d^{\prime}\left(T_{\alpha} x, T_{\alpha} y\right) \mid \alpha \in A\right\}$ is finite. If $x_{0} \in S \leqq X$, we say $\mathscr{F}$ is uniformly bounded on $S$ if $\sup \left\{d^{\prime}\left(T_{\alpha} x, T_{\alpha}, x_{0}\right) \mid x \in S, \alpha \in A\right\}$ is finite. A uniform boundedness theorem is one in which uniform boundedness (for some family $\mathscr{F}$ ) is deduced from pointwise-boundedness.

Let $\gamma:[0,1] \rightarrow X$ be continuous, $0=t_{0}<\cdots<t_{n}=1$ a partition $P$ of $[0,1]$, define $\ell(\gamma, P)=\sum_{k=1}^{n} d\left(\gamma\left(t_{k}\right), \gamma\left(t_{k-1}\right)\right)$, and define $\ell(\gamma)$ to be the supremum over all partitions $P$ of the $\ell(\gamma, P)$. For $x, y \in X$, define $d_{g}(x, y)=\inf \{\ell(\gamma) \mid \gamma:[0,1] \rightarrow X, \gamma(0)=x, \gamma(1)=y\}$; this is the geodesic or intrinsic distance between $x$ and $y \cdot d_{g}$ is a generalized metric, and $\gamma$ is said to be a segment from $x$ to $y$ if

$$
\gamma(0)=x, \gamma(1)=y,
$$

and $\ell(\gamma)=d_{g}(x, y)<\infty$.

Definition 1. $X$ is said to be geodesically convex if for any $x, y$ in $X$ there is a unique segment from $x$ to $y$. We denote by $\Phi_{g}(x, y, t)$ the intrinsic parametrization of this segment (if $0 \leqq t \leqq$ $s \leqq 1, d_{g}\left(\Phi_{g}(x, y, t), \Phi_{g}(x, y, s)\right)=(s-t) d_{g}(x, y) ; T$ is said to be an affine map between geodesically convex spaces if $T\left(\Phi_{g}(x, y, t)\right)=\Phi_{g}(T x, T y, t)$.

A term often used for a geodesically convex space is a space with 
unique segments. Throughout this paper we assume $d=d_{g}$.

Our first theorem is a generalization to geodesically convex spaces of the classical Banach-Steinhaus Theorem.

TheOrem 1. Let $(X, d)$ be a geodesically convex complete metric space and let $\left(Y, d^{\prime}\right)$ be geodesically convex. Let $\mathscr{F}=\left\{T_{\alpha} \mid \alpha \in A\right\}$ be a pointwise-bounded family of geodesically affine maps from $X$ to $Y$, each of which is continuous. Then for each $x_{0} \in X$,

$$
\sup \left\{d^{\prime}\left(T_{\alpha} x, T_{\alpha} x_{0}\right) \mid \alpha \in A, d\left(x, x_{0}\right) \leqq 1\right\}
$$

is finite.

We shall need the following lemma.

Lemma 1. For each $\alpha \in A, z_{0} \in X$ and $p>0$,

$$
r\left(\alpha, z_{0}, p\right)=\sup \left\{d^{\prime}\left(T_{\alpha} x, T_{\alpha} z_{0}\right) \mid d\left(x, z_{0}\right) \leqq p\right\}
$$

is finite.

Proof. By continuity of $T_{\alpha}$ at $z_{0}, \exists \delta>0$ such that

$$
d\left(x, z_{0}\right)<\delta \Rightarrow d^{\prime}\left(T_{\alpha} x, T_{\alpha} z_{0}\right)<1 ;
$$

we can clearly assume $\delta<p$. If $x \in X, d\left(x, z_{0}\right) \leqq p$, let $z=\Phi_{g}\left(z_{0}, x, \delta / 2 p\right)$, then $d\left(z_{0}, z\right)=\delta / 2 p d\left(z_{0}, x\right)<\delta$, so $d^{\prime}\left(T_{\alpha} z_{0}, T_{\alpha} z\right)<1$. But

$$
T_{\alpha}\left(\Phi_{g}\left(z_{0}, x, \delta / 2 p\right)\right)=\Phi_{g}\left(T_{\alpha} z_{0}, T_{\alpha} x, \delta / 2 p\right),
$$

and so $d^{\prime}\left(T_{\alpha} z_{0}, T_{\alpha} z\right)=\delta / 2 p d^{\prime}\left(T_{\alpha} z_{0}, T_{\alpha} x\right)<1$, so $d^{\prime}\left(T_{\alpha} z_{0}, T_{\alpha} x\right)<2 p / \delta$.

For purposes of simplicity, we prove the following lemma.

Lemma 2. Assume the conclusion of the theorem is false. Let $M>0, x_{1}, \cdots, x_{n} \in X$ and $T_{1}, \cdots, T_{n} \in \mathscr{F}$ be given, with $d\left(x_{0}, x_{k}\right)<$ $1(1 \leqq k \leqq n)$. Then $\exists x_{n+1} \in X, T_{n+1} \in \mathscr{F}$ with $d\left(x_{0}, x_{n+1}\right)<1, d\left(x_{n}, x_{n+1}\right)<$ $1 / 2^{n+1}, d^{\prime}\left(T_{n+1} x_{n+1}, T_{n+1} x_{0}\right)>M$, and

$$
d^{\prime}\left(T_{k} x_{n}, T_{k} x_{n+1}\right)<1 / 2^{n+1}
$$

for $1 \leqq k \leqq n$.

Proof. For $x \in X$, let $S(x)=\sup \left\{d^{\prime}\left(T_{\alpha} x, T_{\alpha} x_{0}\right) \mid \alpha \in A\right\}$. Let $\alpha=1 / 3 \min \left(2^{-n-1} r\left(1, x_{n}, 2\right)^{-1}, \cdots, 2^{-n-1} r\left(n, x_{n}, 2\right)^{-1}, 2^{-n-1}, 1-d\left(x_{n}, x_{0}\right)\right)$, then $\alpha>0$. If the theorem is false, then for any $K>0$ there is a $z \in X$ with $d\left(x_{0}, z\right)<1$ and a $T \in \mathscr{F}$ with $K<d^{\prime}\left(T x_{0}, T z\right)$, consequently 


$$
K<d^{\prime}\left(T x_{0}, T z\right) \leqq d^{\prime}\left(T x_{0}, T x_{n}\right)+d^{\prime}\left(T x_{n}, T z\right) \leqq S\left(x_{n}\right)+d^{\prime}\left(T x_{n}, T z\right) .
$$

This means that we can always find a $z \in X$ and $T \in \mathscr{F}$ with

$$
d\left(x_{0}, z\right)<1
$$

and $d^{\prime}\left(T x_{n}, T z\right)$ arbitrarily large. Having defined $\alpha$, choose $y \in X$, $T\left(=T_{n+1}\right) \in \mathscr{F}$ with $d\left(y, x_{0}\right)<1, \alpha d^{\prime}\left(T x_{n}, T y\right)-S\left(x_{n}\right)>M$, and let $x_{n+1}=\Phi_{g}\left(x_{n}, y, \alpha\right)$. Then $d\left(x_{n}, x_{n+1}\right)=\alpha d\left(x_{n}, y\right) \leqq 2^{-n-1}$. For $1 \leqq k \leqq n$, we have

$$
\begin{aligned}
d^{\prime}\left(T_{k} x_{n}, T_{k} x_{n+1}\right) & =d^{\prime}\left(T_{k} \Phi_{g}\left(x_{n}, y, 0\right), T_{k} \Phi_{g}\left(x_{n}, y, \alpha\right)\right) \\
& =d^{\prime}\left(\Phi_{g}\left(T_{k} x_{n}, T_{k} y, 0\right), \Phi_{g}\left(T_{k} x_{n}, T_{k} y, \alpha\right)\right) \\
& =\alpha d^{\prime}\left(T_{k} x_{n}, T_{k} y\right) \leqq \alpha r\left(k, x_{n}, 2\right)<2^{-n-1}
\end{aligned}
$$

We also have

$$
\begin{aligned}
d\left(x_{0}, x_{n+1}\right) & \leqq d\left(x_{0}, x_{n}\right)+d\left(x_{n}, x_{n+1}\right) \\
& \leqq d\left(x_{0}, x_{n}\right)+d\left(\Phi_{g}\left(x_{n}, y, 0\right), \Phi_{g}\left(x_{n}, y, \alpha\right)\right) \\
& =d\left(x_{0}, x_{n}\right)+\alpha d\left(x_{n}, y\right)<d\left(x_{0}, x_{n}\right)+2 \alpha \\
& <d\left(x_{0}, x_{n}\right)+1-d\left(x_{0}, x_{n}\right) \\
& =1 .
\end{aligned}
$$

Finally,

$$
\begin{aligned}
\alpha d^{\prime}\left(T x_{n}, T y\right) & =d^{\prime}\left(T x_{n}, T x_{n+1}\right) \\
& \leqq d^{\prime}\left(T x_{n}, T x_{0}\right)+d\left(T x_{0}, T x_{n+1}\right) \\
& \leqq S\left(x_{n}\right)+d^{\prime}\left(T x_{0}, T x_{n+1}\right) \Longrightarrow d^{\prime}\left(T x_{0}, T x_{n+1}\right) \\
& \geqq \alpha d\left(T x_{n}, T y\right)-S\left(x_{n}\right)>M,
\end{aligned}
$$

completing the proof.

We return to the proof of the theorem. Assume the theorem is false. Then $\exists x_{1} \in X, T_{1} \in \mathscr{F}$ with

$$
d\left(x_{0}, x_{1}\right)<1, d^{\prime}\left(T_{1} x_{0}, T_{1} x_{1}\right)>2 .
$$

Having chosen $x_{1}, \cdots, x_{n} \in X, T_{1}, \cdots, T_{n} \in \mathscr{F}$ with

$$
d\left(x_{0}, x_{k}\right)<1(1 \leqq k \leqq n),
$$

by Lemma 2 choose $x_{n+1} \in X, T_{n+1} \in \mathscr{F}$ with $d\left(x_{0}, x_{n+1}\right)<1, d\left(x_{n}, x_{n+1}\right)<$ $2^{-n-1}, d^{\prime}\left(T_{n+1} x_{0}, T_{n+1} x_{n+1}\right)>n+2$ and $d^{\prime}\left(T_{k} x_{n}, T_{k} x_{n+1}\right)<2^{-n-1}$ for $1 \leqq k \leqq n$. Since $d\left(x_{n}, x_{n+1}\right)<2^{-n-1}$, the sequence $\left\{x_{n} \mid n=1,2, \cdots\right\}$ is Cauchy $\left(n<m \Rightarrow d\left(x_{n}, x_{m}\right)<\sum_{k=n}^{m-1} 2^{-k-1}\right)$; by completeness $x_{n} \rightarrow x \in X$. By continuity of $T_{n}$ we have $\lim _{m \rightarrow \infty} d^{\prime}\left(T_{n} x, T_{n} x_{m+1-1}\right)=0$, so 


$$
\begin{aligned}
d^{\prime}\left(T_{n} x_{0}, T_{n} x_{n}\right) & \leqq d^{\prime}\left(T_{n} x_{0}, T_{n} x\right)+d^{\prime}\left(T_{n} x, T_{n} x_{n}\right) \leqq \cdots \\
& \leqq d^{\prime}\left(T_{n} x_{0}, T_{n} x\right)+\sum_{k=n}^{m} d^{\prime}\left(T_{n} x_{k}, T_{n} x_{k+1}\right)+d^{\prime}\left(T_{n} x, T_{n} x_{m+1}\right) ;
\end{aligned}
$$

letting $m \rightarrow \infty$ we obtain

$$
\begin{aligned}
& d^{\prime}\left(T_{n} x_{0}, T_{n} x_{n}\right) \leqq d^{\prime}\left(T_{n} x_{0}, T_{n} x\right)+\sum_{k=n}^{\infty} d^{\prime}\left(T_{n} x_{k}, T_{n} x_{k+1}\right) \\
& <d^{\prime}\left(T_{n} x_{0}, T_{n} x\right)+\sum_{k=n}^{\infty} 2^{-k-1}<d^{\prime}\left(T_{n} x_{0}, T_{n} x\right)+1,
\end{aligned}
$$

since $k \geqq n \Rightarrow d^{\prime}\left(T_{n} x_{k}, T_{n} x_{k+1}\right)<2^{-k-1}$. So

$$
n+1<d^{\prime}\left(T_{n} x_{0}, T_{n} x_{n}\right) \leqq d^{\prime}\left(T_{n} x_{0}, T_{n} x\right)+1 \Rightarrow d^{\prime}\left(T_{n} x_{0}, T_{n} x\right)>n,
$$

contradicting the pointwise-boundedness of $\mathscr{F}$.

We now make an additional hypothesis, which will enable us to prove a stronger version of this theorem. Let $\Phi=\Phi_{g}$.

Definition 2. If $0<\alpha<1$, define

$$
M(\alpha)=\sup \{d(\Phi(x, y, \alpha), \Phi(x, z, \alpha)) / d(y, z) \mid x, y, z \in X, y \neq z\},
$$

and define $M^{\prime}(\alpha)$ similarly in $Y$. Note that, if $M(\alpha)<\infty$, then

$$
x, y, z \in X \Rightarrow d(\Phi(x, y, \alpha), \Phi(x, z, \alpha)) \leqq M(\alpha) d(y, z) .
$$

For the remainder of this paper we shall make the following assumption: $\exists \alpha \in(0,1)$ such that both $M(\alpha)$ and $M^{\prime}(\alpha)$ are finite. This $\alpha$ will be fixed from now on.

Definition 3. Let $\left\{x_{n} \mid n=1,2, \cdots\right\} \subseteq X$, and let $x_{0} \in X$. Define $\boldsymbol{z}_{1}^{(n)}=\Phi\left(x_{n}, x_{0}, \alpha\right)$, and for $2 \leqq k \leqq n$ define $z_{k}^{(n)}=\Phi\left(x_{n+1-k}, z_{k-1}^{(n)}, \alpha\right)$. Now define $y_{n}=z_{n}^{(n)}$ for $n=1,2, \cdots$.

If $X$ were a Banach space and $x_{0}=0$, then we would have

$$
y_{n}=\sum_{k=1}^{n}(1-\alpha)^{k} x_{k}
$$

In general, however, we have $y_{n}=\Phi\left(x_{1}, \Phi\left(x_{2}, \cdots, \Phi\left(x_{n}, x_{0}, \alpha\right), \cdots, \alpha\right)\right.$, which will henceforth be abbreviated $\Phi\left(x_{1}, \cdots, \Phi\left(x_{n}, x_{0}, \alpha\right), \cdots, \alpha\right)$.

Lemma 3. Given

$$
\left\{a_{n} \mid n=1,2, \cdots\right\} \subseteq X,
$$

$x_{0} \in X$, define $\left\{y_{n} \mid n=1,2, \cdots\right\}$ as in Definition 3. Then 


$$
d\left(y_{n}, y_{n-1}\right) \leqq M(\alpha)^{n-1}(1-\alpha) d\left(x_{n}, x_{0}\right)
$$

if $n \geqq 2$.

Proof. Clearly, we have

$$
\begin{aligned}
& d\left(y_{n}, y_{n-1}\right) \\
= & d\left(\Phi\left(x_{1}, \cdots, \Phi\left(x_{n}, x_{0}, \alpha\right), \cdots, \alpha\right), \Phi\left(x_{1}, \cdots, \Phi\left(x_{n-1}, x_{0}, \alpha\right), \cdots, \alpha\right)\right) \\
\leqq & M(\alpha) d\left(\Phi\left(x_{2}, \cdots, \Phi\left(x_{n}, x_{0}, \alpha\right), \cdots, \alpha\right), \Phi\left(x_{2}, \cdots, \Phi\left(x_{n-1}, x_{0}, \alpha\right), \cdots, \alpha\right)\right) \\
\leqq & \cdots \leqq M(\alpha)^{n-2} d\left(\Phi\left(x_{n-1}, \Phi\left(x_{n}, x_{0}, \alpha\right), \alpha\right), \Phi\left(x_{n-1}, x_{0}, \alpha\right)\right) \\
\leqq & M(\alpha)^{n-1} d\left(\Phi\left(x_{n}, x_{0}, \alpha\right), x_{0}\right) \\
= & (1-\alpha) M(\alpha)^{n-1} d\left(x_{n}, x_{0}\right) .
\end{aligned}
$$

LEMma 4. Let $S$ be a convex subset of $X, p>0$, and let $x_{0} \in S$, $\mathscr{F}=\left\{T_{\lambda} \mid \lambda \in \Lambda\right\}$ a collection of affine functions on $X$. If $\mathscr{F}$ is not uniformly bounded on $S \cap S\left(x_{0}, p\right)$, then given $M>0, \varepsilon>0$, we can find a $T \in \mathscr{F}$ and an $x \in S \cap S\left(x_{0}, p\right)$ such that $d\left(x_{0}, x\right)<\varepsilon$ and

$$
d^{\prime}\left(T x, T x_{0}\right)>M
$$

Proof. We can assume without loss of generality that $\varepsilon<p$. Choose $T \in \mathscr{F}, y \in S \cap S\left(x_{0}, p\right)$ such that $d^{\prime}\left(T y, T x_{0}\right)>M p / \varepsilon$. Let $x=$ $\Phi\left(x_{0}, y, \varepsilon / p\right) ; x \in S$ by the convexity of $S$. Now

$$
d\left(x, x_{0}\right)=(\varepsilon / p) d\left(y, x_{0}\right)<\varepsilon,
$$

and

$$
\begin{aligned}
d^{\prime}\left(T x, T x_{0}\right) & =d^{\prime}\left(T \Phi\left(x_{0}, y, \varepsilon / p\right), T x_{0}\right) \\
& =d^{\prime}\left(\Phi\left(T x_{0}, T y, \varepsilon / p\right), T x_{0}\right) \\
& =\varepsilon / p d^{\prime}\left(T x_{0}, T y\right)>M,
\end{aligned}
$$

completing the proof.

The next lemma will be critical in proving the desired theorem.

LEMMA 5. Let $\left\{S_{n} \mid n=1,2, \cdots\right\}$ be a collection of closed convex subsets of $X$, and let $\left\{T_{n} \mid n=1,2, \cdots\right\}$ be a collection of affine functions on $X$ such that $T_{n} \mid S_{n}$ is continuous for $n=1,2, \cdots$. Assume that $x_{n+1} \in \bigcap_{k=1}^{n} S_{k}$ for $n=1,2, \cdots$, and that $d\left(x_{n}, x_{0}\right)$ is sufficiently small to make $\left\{y_{n} \mid n=1,2, \cdots\right\}$ (as defined in Definition 2) a Cauchy sequence (we do this by requiring $\sum_{n=1}^{\infty} M(\alpha)^{n-1} d\left(x_{n}, x_{0}\right)$ to converge). By completeness of $X$, let $y=\lim _{n \rightarrow \infty} y_{n}$. Then for each integer $N$, $T_{N} y=\lim _{n \rightarrow \infty} T_{N} y_{n}$. 
Proof. Observe first that, if $\lim _{n \rightarrow \infty} u_{n}=u$ (in either $X$ or $Y$ ), then $\lim _{n \rightarrow \infty} \Phi\left(v, u_{n}, \alpha\right)=\Phi(v, u, \alpha)$, as

$$
d\left(\Phi\left(v, u_{n}, \alpha\right), \Phi(v, u, \alpha)\right) \leqq M(\alpha) d\left(u_{n}, u\right) \rightarrow 0 .
$$

If $n>N$, let $z_{n}=\Phi\left(x_{N+1}, \cdots, \Phi\left(x_{n}, x_{0}, \alpha\right), \cdots, \alpha\right)$. As in Lemma 1 , we can show that $d\left(z_{n}, z_{n-1}\right) \leqq(1-\alpha) M(\alpha)^{n-N-1} d\left(x_{n}, x_{0}\right)$, and since $\sum_{n=1}^{\infty} M(\alpha)^{n-1} d\left(x_{n}, x_{0}\right)$ converges, we can define $z=\lim _{n \rightarrow \infty} z_{n}$. Note that $n>N \Rightarrow z_{n} \in S_{N}$, as $x_{N+1}, \cdots, x_{n} \in S_{N}$ and $S_{N}$ is convex. Since $S_{N}$ is closed, $z \in S_{N}$, and so $T_{N} z_{n} \rightarrow T_{N} z$ by the continuity of $T_{N} \mid S_{N}$. If $n>N$, we have

$$
\begin{aligned}
T_{N} y_{n} & =T_{N} \Phi\left(x_{1}, \cdots, \Phi\left(x_{N}, z_{n}, \alpha\right), \cdots, \alpha\right) \\
& =\Phi\left(T_{N} x_{1}, \cdots, \Phi\left(T_{N} x_{N}, T_{N} z_{n}, \alpha\right), \cdots, \alpha\right),
\end{aligned}
$$

and so

$$
\begin{aligned}
\lim _{n \rightarrow \infty} T_{N} y_{n} & =\Phi\left(T_{N} x_{1}, \cdots, \lim _{n \rightarrow \infty} \Phi\left(T_{N} x_{N}, T_{N} z_{n}, \alpha\right), \cdots, \alpha\right) \\
& =\Phi\left(T_{N} x_{1}, \cdots, \Phi\left(T_{N} x_{N}, \lim _{n \rightarrow \infty} T_{N} z_{n}, \alpha\right), \cdots, \alpha\right) \\
& =\Phi\left(T_{N} x_{1}, \cdots, \Phi\left(T_{N} x_{N}, T_{N} z, \alpha\right), \cdots, \alpha\right) .
\end{aligned}
$$

Since $y_{n}=\Phi\left(x_{1}, \cdots, \Phi\left(x_{N}, z_{n}, \alpha\right), \cdots, \alpha\right)$ and

$$
\begin{aligned}
y & =\lim _{n \rightarrow \infty} y_{n}=\Phi\left(x_{1}, \cdots, \lim _{n \rightarrow \infty} \Phi\left(x_{N}, z_{n}, \alpha\right)\right) \\
& =\Phi\left(x_{1}, \cdots, \Phi\left(x_{N}, z, \alpha\right), \cdots, \alpha\right),
\end{aligned}
$$

we see that $T_{N} y=\Phi\left(T_{N} x_{1}, \cdots, \Phi\left(T_{N} x_{N}, T_{N} z, \alpha\right), \cdots, \alpha\right)=\lim _{n \rightarrow \infty} T_{N} y_{n}$.

It is now necessary to perform some calculations. Assume

$$
\left\{x_{n} \mid n=1,2, \cdots\right\} \subseteq X,
$$

$x_{0} \in X$, and $\left\{y_{n} \mid n=1,2, \cdots\right\}$ is defined as in Definition 3. Now define

$$
z_{k}=\Phi\left(x_{k}, \cdots, \Phi\left(x_{n}, x_{0}, \alpha\right), \cdots, \alpha\right)=\Phi\left(x_{k}, z_{k+1}, \alpha\right)
$$

(for the purpose of these calculations, $n$ will be assumed to be fixed) for $k \leqq n-1, z_{n}=\Phi\left(x_{n}, x_{0}, \alpha\right)$. We now have $d\left(x_{0}, \Phi\left(x_{n}, x_{0}, \alpha\right)\right)=$ $d\left(x_{0}, z_{n}\right) \leqq d\left(x_{0}, y_{n}\right)+\sum_{k=1}^{n-1}\left(d\left(z_{k}, z_{k+1}\right)\right.$, as clearly $z_{1}=y_{n}$. Observe further that

$$
\begin{aligned}
d\left(z_{k}, z_{k+1}\right) & =d\left(\Phi\left(x_{k}, z_{k+1}, \alpha\right), z_{k+1}\right) \\
& =(1-\alpha) d\left(x_{k}, z_{k+1}\right) \\
& \leqq(1-\alpha)\left[d\left(x_{k}, x_{0}\right)+d\left(x_{0}, z_{k+1}\right)\right]
\end{aligned}
$$

for $\mathrm{k} \leqq n-1$. 
We now prove some computational lemmas.

LEMMA 6. If $k \leqq n-2$,

$$
d\left(x_{0}, z_{k+1}\right) \leqq(1+\alpha) d\left(x_{k+1}, x_{0}\right)+\alpha d\left(x_{0}, z_{k+2}\right) .
$$

Proof.

$$
\begin{aligned}
d\left(x_{0},\right. & \left.z_{k+1}\right) \leqq d\left(z_{k+1}, x_{k+1}\right)+d\left(x_{k+1}, x_{0}\right) \\
& =d\left(\Phi\left(x_{k+1}, z_{k+2}, \alpha\right), x_{k+1}\right)+d\left(x_{k+1}, x_{0}\right) \\
& =\alpha d\left(x_{k+1}, z_{k+2}\right)+d\left(x_{k+1}, x_{0}\right) \\
& \leqq \alpha\left[d\left(x_{k+1}, x_{0}\right)+d\left(x_{0}, z_{k+2}\right)\right]+d\left(x_{k+1}, x_{0}\right) \\
& =(1+\alpha) d\left(x_{k+1}, x_{0}\right)+\alpha d\left(x_{0}, z_{k+2}\right) .
\end{aligned}
$$

LEMma 7. If $k \leqq n-2$, then

$$
d\left(x_{0}, z_{k+1}\right) \leqq(1+\alpha) \sum_{j=0}^{n-k-2} \alpha^{j} d\left(x_{0}, x_{k+1+j}\right)+(1-\alpha)^{n-k-1} d\left(x_{0}, x_{n}\right) .
$$

Proof. If $j \leqq n-k-2$, we shall verify the inequality

$$
d\left(x_{0}, z_{k+1}\right) \leqq(1+\alpha) \sum_{i=0}^{j} \alpha^{i} d\left(x_{0}, x_{k+1+i}\right)+\alpha^{j+1} d\left(x_{0}, z_{k+j+2}\right) .
$$

If $j=0$, this inequality is the conclusion of Lemma 4. Inductively, assume it is true for $j$. By Lemma 6 , we have

$$
\alpha^{j+1} d\left(x_{0}, z_{k+j+2}\right) \leqq \alpha^{j+1}\left[(1+\alpha) d\left(x_{0}, x_{k+j+2}\right)+\alpha d\left(x_{0}, z_{k+j+3}\right)\right] ;
$$

adding this term to the $j^{\text {th }}$ inequality yields the inequality for $j+1$. When $j=n-k-2$, we therefore have

$$
\begin{aligned}
d\left(x_{0}, z_{k+1}\right) & \leqq \sum_{j=0}^{n-k-2}(1+\alpha) \alpha^{j} d\left(x_{0}, x_{k+1+j}\right)+\alpha^{n-k-1} d\left(x_{0}, z_{n}\right) \\
& =(1+\alpha) \sum_{j=0}^{n-k-2} \alpha^{j} d\left(x_{0}, x_{k+1+j}\right)+(1-\alpha) \alpha^{n-k-1} d\left(x_{0}, x_{n}\right) .
\end{aligned}
$$

A consequence of Lemma 7 and a previous observation is that

$$
\begin{aligned}
d\left(z_{k}, z_{k+1}\right) \leqq & (1-\alpha)\left[d\left(x_{k}, x_{0}\right)+d\left(x_{0}, z_{k+1}\right)\right] \\
\leqq & (1-\alpha)\left[d\left(x_{k}, x_{0}\right)+(1+\alpha) \sum_{j=0}^{n-k-2} \alpha^{j} d\left(x_{0}, x_{k+1+j}\right)\right. \\
& \left.+(1-\alpha) \alpha^{n-k-1} d\left(x_{0}, x_{n}\right)\right] .
\end{aligned}
$$

Now let $1 \leqq k \leqq n-1$. We make the following definition for $k \leqq j \leqq n$.

$$
\begin{aligned}
\mu_{j}^{(k)} & =1-\alpha & & \text { if } j=k \\
& =\left(1-\alpha^{2}\right) \alpha^{j-k-1} & & \text { if } k<j<n \\
& =(1-\alpha)^{2} \alpha^{n-k-1} & & \text { if } j=n .
\end{aligned}
$$


Then $d\left(z_{k}, z_{k+1}\right) \leqq \sum_{j=k}^{n} \mu_{j}^{(k)} d\left(x_{j}, x_{0}\right)$, and so

$$
\begin{aligned}
(1-\alpha) d\left(x_{0}, x_{n}\right) & =d\left(x_{0}, \Phi\left(x_{n}, x_{0}, \alpha\right)\right) \\
& \leqq d\left(x_{0}, y_{n}\right)+\sum_{k=1}^{n-1} d\left(z_{k}, z_{k+1}\right) \\
& \leqq d\left(x_{0}, y_{n}\right)+\sum_{k=1}^{n-1}\left(\sum_{j=k}^{n} \mu_{j}^{(k)} d\left(x_{j}, x_{0}\right)\right) \\
& =d\left(x_{0}, y_{n}\right)+\sum_{k=1}^{n-1}\left(\sum_{j=1}^{k} \mu_{k}^{(j)}\right) d\left(x_{k}, x_{0}\right)+\sum_{j=1}^{n-1} \mu_{n}^{(j)} d\left(x_{n}, x_{0}\right) .
\end{aligned}
$$

If $1 \leqq k \leqq n-1$, let $\beta_{k}=\sum_{\jmath=1}^{k} \mu_{k}^{(j)}$, and let

$$
\beta_{n}=\sum_{j=1}^{n-1} \mu_{n}^{(j)}-(1-\alpha) \text {. }
$$

Obviously $\beta_{k}>0$ if $1 \leqq k \leqq n-1$, and also

$$
\begin{aligned}
\sum_{j=1}^{n-1} \mu_{n}^{(j)} & =(1-\alpha)^{2} \sum_{j=1}^{n-1} \alpha^{n-j-1} \\
& =(1-\alpha)^{2} \sum_{j=0}^{n-2} \alpha^{j} \\
& =(1-\alpha)^{2}\left[\left(1-\alpha^{n-1}\right) /(1-\alpha)\right] \\
& =(1-\alpha)\left(1-\alpha^{n-1}\right)<1-\alpha,
\end{aligned}
$$

and so $\beta_{n}<0$. Since this calculation has been performed for the integer $n$, we shall relabel the constants just obtained $\beta_{1}^{(n)}, \cdots, \beta_{n}^{(n)}$.

The last inequality proved shows that

$$
0 \leqq d\left(x_{0}, y_{n}\right)+\sum_{k=1}^{n} \beta_{k}^{(n)} d\left(x_{0}, x_{k}\right),
$$

which implies that $d\left(x_{0}, y_{n}\right) \geqq\left(-\beta_{n}^{(n)}\right) d\left(x_{0}, x_{n}\right)-\sum_{k=1}^{n-1} \beta_{k}^{(n)} d\left(x_{0}, x_{k}\right)$. A reexamination of the work done subsequent to Lemma 3 shows that, if $T: X \rightarrow Y$ is affine, then

$$
d^{\prime}\left(T x_{0}, T y_{n}\right) \geqq\left(-\beta_{n}^{(n)}\right) d^{\prime}\left(T x_{0}, T x_{n}\right)-\sum_{k=1}^{n-1} \beta_{k}^{(n)} d^{\prime}\left(T x_{0}, T x_{k}\right) .
$$

We have therefore proved the following:

Lemma 8. Let $\mathscr{F}=\left\{T_{\lambda} \mid \lambda \in \Lambda\right\}$ be a pointwise-bounded family of affine functions from $X$ into $Y$, and let $\left\{x_{n} \mid n=1,2, \cdots\right\}$ be given in $X,\left\{y_{n} \mid n=1,2, \cdots\right\}$ as in Definition 2. If

$$
\left.S(x)=\sup d^{\prime}\left(T x, T x_{0}\right) \mid T \in \mathscr{F}\right\},
$$

then $d^{\prime}\left(T x_{0}, T y_{n}\right) \geqq\left(-\beta_{n}^{(n)}\right) d^{\prime}\left(T x_{0}, T x_{n}\right)-\sum_{k=1}^{n-1} \beta_{k}^{(n)} S\left(x_{k}\right)$ for any $T \in \mathscr{F}$.

Proof. Immediate from previous work and the fact that 


$$
d^{\prime}\left(T x_{0}, T x_{k}\right) \leqq S\left(x_{k}\right)
$$

for all $T \in \mathscr{F}$.

We come now to the desired theorem.

THEOREM 2. Let $(X, d),\left(Y, d^{\prime}\right)$ be spaces with unique segments, let $X$ be complete, and assume there is an $\alpha \in(0,1)$ such that $M(\alpha)$, $M^{\prime}(\alpha)$ are finite. Let $\mathscr{F}=\left\{T_{\lambda} \mid \lambda \in \Lambda\right\}$ be a pointwise-bounded family of affine maps from $X$ into $Y$, and let $S_{\lambda}$ be a closed convex subset of $X$ such that $\bigcap_{\lambda \in \Lambda} S_{\lambda} \neq \varphi$ and $T_{\lambda} \mid S_{\lambda}$ is continuous for each $\lambda \in \Lambda$. Then $\exists \lambda_{1}, \cdots, \lambda_{n} \in \Lambda$ such that $\mathscr{F}$ is uniformly bounded on $\bigcap_{k=1}^{n} S_{\lambda_{k}}$.

Proof. Let $x_{0} \in \bigcap_{\lambda \in \Lambda} S_{\lambda}, p>0$, and assume that $\mathscr{F}$ is not uniformly bounded on the intersection of $S\left(x_{0}, p\right)$ and any finite intersection of the $\left\{S_{\lambda} \mid \lambda \in \Lambda\right\}$. We assert that we can prove the following: given $x_{1}, \cdots, x_{n} \in X, T_{1}, \cdots, T_{n} \in \mathscr{F}$ with $T_{k} \mid S_{k}$ continuous, $1 \leqq k \leqq n$ and $x_{k} \in \bigcap_{j=1}^{k-1} S_{j}$ for $2 \leqq k \leqq n$, and given $M>0$, let $y_{1}, \cdots, y_{n}$ be derived from $x_{1}, \cdots, x_{n}$ as in Definition 3. Then we can find $x_{n+1} \in$ $\bigcap_{k=1}^{n} S_{k}$ and $T_{n+1} \in \mathscr{F}$ such that, if we let $y_{n+1}$ be derived from $x_{1}, \cdots, x_{n+1}$ as in Definition 3 ,

$$
d\left(x_{0}, y_{n+1}\right)<p, d\left(y_{n}, y_{n+1}\right)<1 / 2^{n+1}, d^{\prime}\left(T_{n+1} y_{n+1}, T_{n+1} x_{0}\right)>M,
$$

and $d^{\prime}\left(T_{k} y_{n}, T_{k} y_{n+1}\right)<1 / 2^{n+1}$ for $1 \leqq k \leqq n$.

Since $x_{0} \in \bigcap_{k=1}^{n} S_{k}$, choose $\delta_{k}(1 \leqq k \leqq n)$ such that $x \in S_{k}$,

$$
d\left(x, x_{0}\right)<\delta_{k} \Rightarrow d^{\prime}\left(T_{k} x, T_{k} x_{0}\right)<1 / 2^{n+1}(1-\alpha) M^{\prime}(\alpha)^{n} ;
$$

then if we define $y=\Phi\left(x_{1}, \cdots, \Phi\left(x_{n}, \Phi\left(x, x_{0}, \alpha\right), \alpha\right), \cdots, \alpha\right)$, by Lemma 3 we have $x \in S_{k}, d\left(x, x_{0}\right)<\delta_{k} \Rightarrow d^{\prime}\left(T_{k} y_{n}, T_{k} y\right)<1 / 2^{n+1}$. Now let

$\gamma=2^{-1} \min \left(p, \delta_{1}, \cdots, \delta_{n},\left(p-d\left(x_{0}, y_{n}\right)\right) /(1-\alpha) M(\alpha)^{n}, 1 /(1-\alpha) M(\alpha)^{n} 2^{n+1}\right)$.

Finally, by Lemma 4 choose $x_{n+1} \in \bigcap_{k=1}^{n} S_{k}$ and $T\left(=T_{n+1}\right) \in \mathscr{F}$ with $d\left(x_{0}, x_{n+1}\right)<\gamma$ and $\left(-\beta_{n+1}^{(n+1)}\right) d^{\prime}\left(T x_{0}, T x_{n+1}\right)>M+\sum_{k=1}^{n} \beta_{k}^{(n+1)} S\left(x_{k}\right)$. Define $y_{n+1}=\Phi\left(x_{1}, \cdots, \Phi\left(x_{n+1}, x_{0}, \alpha\right), \cdots, \alpha\right)$. We have already observed that $1 \leqq k \leqq n \Rightarrow d^{\prime}\left(T_{k} y_{n}, T_{k} y_{n+1}\right)<1 / 2^{n+1}$. Now by Lemma 3

$$
d\left(y_{n}, y_{n+1}\right) \leqq(1-\alpha) M(\alpha)^{n} d\left(x_{0}, x_{n+1}\right)<1 / 2^{n+1},
$$

and also

$$
\begin{aligned}
d\left(x_{0}, y_{n+1}\right) & \leqq d\left(x_{0}, y_{n}\right)+d\left(y_{n}, y_{n+1}\right) \\
& \leqq d\left(x_{0}, y_{n}\right)+(1-\alpha) M(\alpha)^{n} d\left(x_{0}, x_{n+1}\right) \\
& <d\left(x_{0}, y_{n}\right)+\left(p-d\left(x_{0}, y_{n}\right)\right) \\
& =p .
\end{aligned}
$$

By Lemma 8 we see that 


$$
d^{\prime}\left(T y_{n+1}, T x_{0}\right) \geqq\left(-\beta_{n+1}^{(n+1)}\right) d^{\prime}\left(T x_{0}, T x_{n+1}\right)-\sum_{k=1}^{n} \beta_{k}^{(n+1)} S\left(x_{k}\right)>M .
$$

Construct $\left\{y_{n} \mid n=1,2, \cdots\right\}$ by this procedure to insure that

$$
d\left(x_{0}, y_{n+1}\right)<p, d\left(y_{n}, y_{n+1}\right)<1 / 2^{n+1}
$$

and choose $\left\{T_{n} \mid n=1,2, \cdots\right\} \subseteq \mathscr{F}$ with $d^{\prime}\left(T_{n+1} y_{n+1}, T_{n+1} x_{0}\right)>n+2$ and $d^{\prime}\left(T_{k} y_{n}, T_{k} y_{n+1}\right)<1 / 2^{n+1}$ for $1 \leqq k \leqq n$. Now $\left\{y_{n} \mid n=1,2, \cdots\right\}$ is Cauchy, so let $y=\lim _{k \rightarrow \infty} y_{n}$. By Lemma 5 , for each integer $n$ we have

$$
T_{n} y=\lim _{n \rightarrow \infty} T_{n} y_{k},
$$

and so far any $n$ we have $\lim _{m \rightarrow \infty} d^{\prime}\left(T_{n} y, T_{n} y_{m+1}\right)=0$. So

$$
\begin{aligned}
d^{\prime}\left(T_{n} x_{0}, T_{n} y_{n}\right) & \leqq d^{\prime}\left(T_{n} x_{0}, T_{n} y\right)+d^{\prime}\left(T_{n} y, T_{n} y_{n}\right) \leqq \cdots \\
& \leqq d^{\prime \prime}\left(T_{n} x_{0}, T_{n} y\right)+\sum_{k=n}^{m} d^{\prime}\left(T_{n} y_{k}, T_{n} y_{k+1}\right)+d^{\prime}\left(T_{n} y, T_{n} y_{m+1}\right) ;
\end{aligned}
$$

as $m \rightarrow \infty$ we obtain

$$
\begin{aligned}
d^{\prime}\left(T_{n} x_{0}, T_{n} y_{n}\right) & \leqq d^{\prime}\left(T_{n} x_{0}, T_{n} y\right)+\sum_{k=n}^{\infty} d^{\prime}\left(T_{n} y_{k}, T_{n} y_{k+1}\right) \\
& <d^{\prime}\left(T_{n} x_{0}, T_{n} y\right)+\sum_{k=n}^{\infty} 2^{-k-1} \\
& <d^{\prime}\left(T_{n} x_{0}, T_{n} y\right)+1
\end{aligned}
$$

since $k \geqq n \Rightarrow d^{\prime}\left(T_{n} y_{k}, T_{n} y_{k+1}\right)<1 / 2^{k+1}$. So

$$
x+1<d^{\prime}\left(T_{n} x_{0}, T_{n} y_{n}\right) \leqq d^{\prime}\left(T_{n} x_{0}, T_{n} y\right)+1 \Rightarrow d^{\prime}\left(T_{n} x_{0}, T_{n} y\right)>n,
$$

contradicting the pointwise-boundedness of $\mathscr{F}$ and completing the proof.

In conclusion, although spaces such that $M(\alpha)$ is infinite for every $\alpha \in(0,1)$ are highly pathological, it would be nice to know whether or not the restriction that some $M(\alpha)$ and $M^{\prime}(\alpha)$ be finite can be removed.

\section{REFERENCES}

1. G. Choquet, Topology, New York, Academic Press, 1966.

2. N. Dunford and J. Schwartz, Linear Operators, New York, Interscience, 1958.

Received March 17, 1970, and in revised form April 14, 1971. The preparation of this paper was sponsored in part by the U.S. Army Research Office under Grant DA31-124-ARO(D)-355. Reproduction in whole or in part is permitted for any purpose of the United States Government.

University of California, Los Angeles

AND

Århus University, Arhus, Denmark 


\section{PACIFIC JOURNAL OF MATHEMATICS}

\section{EDITORS}

H. SAMELSON

Stanford University

Stanford, California 94305

C. R. Hовву

University of Washington

Seattle, Washington 98105
J. DUGUNDJI

Department of Mathematics

University of Southern California

Los Angeles, California 90007

RICHARD ARENS

University of California

Los Angeles, California 90024

\section{ASSOCIATE EDITORS}
E. F. BECKENBACH
B. H. NeumanN
F. WOLF
K. YoSHIDA

\section{SUPPORTING INSTITUTIONS}

UNIVERSITY OF BRITISH COLUMBIA

CALIFORNIA INSTITUTE OF TECHNOLOGY

UNIVERSITY OF CALIFORNIA

MONTANA STATE UNIVERSITY

UNIVERSITY OF NEVADA

NEW MEXICO STATE UNIVERSITY

OREGON STATE UNIVERSITY

UNIVERSITY OF OREGON

OSAKA UNIVERSITY

UNIVERSITY OF SOUTHERN CALIFORNIA
STANFORD UNIVERSITY

UNIVERSITY OF TOKYO

UNIVERSITY OF UTAH

WASHINGTON STATE UNIVERSITY

UNIVERSITY OF WASHINGTON

AMERICAN MATHEMATICAL SOCIETY CHEVRON RESEARCH CORPORATION NAVAL WEAPONS CENTER 


\section{Pacific Journal of Mathematics}

\section{Vol. 38, No. $1 \quad$ March, 1971}

Bruce Alan Barnes, Banach algebras which are ideals in a Banach algebra ..... 1

David W. Boyd, Inequalities for positive integral operators............... 9

Lawrence Gerald Brown, Note on the open mapping theorem .............. 25

Stephen Daniel Comer, Representations by algebras of sections over Boolean

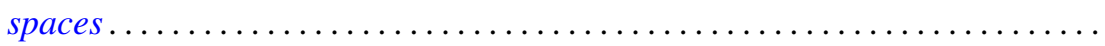

John R. Edwards and Stanley G. Wayment, On the nonequivalence of

conservative Hausdorff methods and Hausdorff moment sequences ........

P. D. T. A. Elliott, On the limiting distribution of additive functions $(\bmod 1) \ldots \ldots$

Mary Rodriguez Embry, Classifying special operators by means of subsets

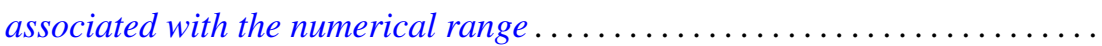

Darald Joe Hartfiel, Counterexamples to a conjecture of G. N. de Oliveira ......

C. Ward Henson, A family of countable homogeneous graphs...............

Satoru Igari and Shigehiko Kuratsubo, A sufficient condition for

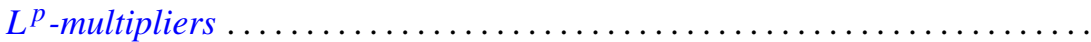

William A. Kirk, Fixed point theorems for nonlinear nonexpansive and

generalized contraction mappings............................

Erwin Kleinfeld, A generalization of commutative and associative rings ...... 95

D. B. Lahiri, Some restricted partition functions. Congruences modulo $11 \ldots \ldots 103$

T. Y. Lin, Homological algebra of stable homotopy ring $\pi *$ of spheres ....... 117

Morris Marden, A representation for the logarithmic derivative of a meromorphic function...........................

John Charles Nichols and James C. Smith, Examples concerning sum properties for metric-dependent dimension functions . .

Asit Baran Raha, On completely Hausdorff-completion of a completely

Hausdorff space.

M. Rajagopalan and Bertram Manuel Schreiber, Ergodic automorphisms and affine transformations of locally compact groups..........

N. V. Rao and Ashoke Kumar Roy, Linear isometries of some function

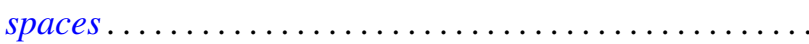

William Francis Reynolds, Blocks and F-class algebras of finite groups

Richard Rochberg, Which linear maps of the disk algebra are multiplicative ...

Gary Sampson, Sharp estimates of convolution transforms in terms of decreasing

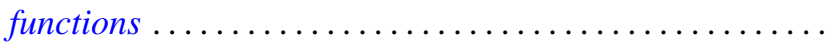

Stephen Scheinberg, Fatou's lemma in normed linear spaces

Ken Shaw, Whittaker constants for entire functions of several complex

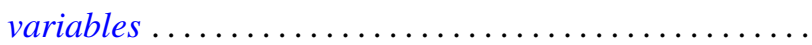

James DeWitt Stein, Two uniform boundedness theorems................ 251

$\mathrm{Li} \mathrm{Pi} \mathrm{Su,} \mathrm{Homomorphisms} \mathrm{of} \mathrm{near-rings} \mathrm{of} \mathrm{continuous} \mathrm{functions} \mathrm{.} \mathrm{.............} 261$

Stephen Willard, Functionally compact spaces, $C$-compact spaces and mappings of minimal Hausdorff spaces....................... 